\title{
Molecular gas in high-velocity clouds: revisited scenario*
}

\author{
M. Dessauges-Zavadsky ${ }^{1}$, F. Combes ${ }^{2}$, and D. Pfenniger ${ }^{1}$ \\ 1 Observatoire de Genève, Université de Genève, 51 Ch. des Maillettes, 1290 Sauverny, Switzerland \\ e-mail: miroslava.dessauges@obs.unige.ch \\ 2 Observatoire de Paris, LERMA, 61 Av. de l'Observatoire, 75014 Paris, France
}

Received 10 February 2007 / Accepted 4 July 2007

\begin{abstract}
We report a new search for ${ }^{12} \mathrm{CO}(1-0)$ emission in high-velocity clouds (HVCs) performed with the IRAM 30 m millimeter-wave telescope. This search was motivated by the recent detection of cold dust emission $(T \sim 10.7 \mathrm{~K})$ in the HVCs of Complex C, implying a total gas column density 5 times larger than the column density measured in H I and suggesting undetected gas, presumably in molecular form. Despite a spatial resolution which is three times better and sensitivity twice as good compared to previous studies, no $\mathrm{CO}$ emission is detected in the HVCs of Complex $\mathrm{C}$ down to a best $5 \sigma$ limit of $0.16 \mathrm{~K} \mathrm{~km} \mathrm{~s}^{-1}$ at a $22^{\prime \prime}$ resolution. The non-detection of both the ${ }^{12} \mathrm{CO}(1-0)$ emission and of the diffuse $\mathrm{H}_{2}$ absorption with the Far Ultraviolet Spectroscopic Explorer does not provide any evidence in favor of large amounts of molecular gas in these HVCs and hence in favor of the infrared findings. We discuss different configurations which, however, allow us to reconcile the negative $\mathrm{CO}$ result with the presence of molecular gas and cold dust emission. $\mathrm{H}_{2}$ column densities higher than our detection limit, $N\left(\mathrm{H}_{2}\right)=3 \times 10^{19} \mathrm{~cm}^{-2}$, are expected to be confined in very small and dense clumps with 20 times smaller sizes than the $0.5 \mathrm{pc}$ clumps resolved in our observations according to the results obtained in cirrus clouds, and might thus still be highly diluted. As a consequence, the inter-clump gas at the $1 \mathrm{pc}$ scale, as resolved in our data, has a volume density lower than $20 \mathrm{~cm}^{-3}$ and already appears as too diffuse to excite the $\mathrm{CO}$ molecules. The observed physical conditions in the HVCs of Complex $\mathrm{C}$ also play an important role against $\mathrm{CO}$ emission detection. The sub-solar metallicity of $0.1-0.3$ dex affects the $\mathrm{H}_{2}$ formation rate onto dust grains, and it has been shown that the CO-to- $\mathrm{H}_{2}$ conversion factor in low metallicity media is 60 times higher than at the solar metallicity, leading for a given $\mathrm{H}_{2}$ column density to a 60 times weaker integrated $\mathrm{CO}$ intensity. And the very low dust temperature estimated in these HVCs implies the possible presence of gas cold enough $(<20 \mathrm{~K})$ to cause CO condensation onto dust grains under interstellar medium pressure conditions and thus $\mathrm{CO}$ depletion in gas-phase observations.
\end{abstract}

Key words. Galaxy: halo - ISM: clouds - ISM: molecules - radio lines: ISM

\section{Introduction}

A meaningful definition of intermediate-velocity clouds (IVCs) and high-velocity clouds (HVCs) is hard to establish. Generally speaking, these neutral gas clouds of the Milky Way halo refer to dynamically significant gas moving several kiloparsecs above the Galactic plane with intermediate $\left(30 \leq\left|v_{\text {LSR }}\right| \leq 90 \mathrm{~km} \mathrm{~s}^{-1}\right)$ and high $\left(\left|v_{\mathrm{LSR}}\right| \geq 90 \mathrm{~km} \mathrm{~s}^{-1}\right)$ radial velocities, respectively. Such radial velocities cannot be ascribed to any reasonable model of differential Galactic rotation. IVCs and HVCs are found in large complexes with angular sizes of 10-90 degrees and cover 10-37\% of the sky (Murphy et al. 1995; Hartmann \& Burton 1997). Since their discovery in $21 \mathrm{~cm}$ observations by Muller et al. (1963), HVCs have been the target of numerous studies (see Wakker \& van Woerden 1997; van Woerden et al. 2004, for a review), and it became rapidly clear that HVCs may have significant implications on the Galaxy as well as on more general issues related to Galactic formation and evolution and to the intergalactic medium (IGM). Thus, the knowledge of both IVC and $\mathrm{HVC}$ nature or origin may lead to fundamental insights.

The origin of IVCs and HVCs, however, is still a matter of debate. The difficulty in determining their distances and metallicities is the major obstacle to pining down their origin. Indeed, the values of many of the physical parameters that can be derived from observations are proportional

$\star$ Based on observations collected with the IRAM $30 \mathrm{~m}$ telescope at Pico Veleta, Spain, in June 20-23, 2006. to a power of the distance (e.g. mass $\propto D^{2}$, size $\propto D$, volume density $\propto D^{-1}$ ), and the metallicity provides indications on a Galactic or extragalactic origin. Recent studies with the Far Ultraviolet Spectroscopic Explorer (FUSE) and the Space Telescope Imaging Spectrograph (STIS) tend to show that the chemical composition of IVCs and HVCs is not uniform, with metallicities varying from 0.1 to 1.0 solar (Wakker et al. 1999; Wakker 2001; Richter et al. 2001a,c, 2003a; Gibson et al. 2000, 2001; Collins et al. 2003, 2007). This strongly suggests that the Galactic halo cloud phenomenon is diverse and can be separated into three different categories.

First, some HVC complexes are proven to be associated with the Milky Way in lying only a few kpc from the disk due to absorptions detected in front of Galactic halo stars (e.g. van Woerden et al. 1999) and having near-solar metallicities. This supports a Galactic origin and more specifically the "Galactic fountain" model where gas is ejected in the halo by the star formation feedback mechanisms (Shapiro \& Field 1976; Houck \& Bregman 1990). Second, some HVCs result from the gas left over from the formation of the Milky Way (Oort 1970). They are dynamically associated with nearby galaxies and are now raining down to the Galactic disk. This is the case, for example, of the dominant HVC feature, the so-called Magellanic Stream, at $\sim 50 \mathrm{kpc}$ and with a metallicity between the Small and Large Magellanic Clouds ( 0.3 solar, Lu et al. 1998), which was identified as tidal debris from the interaction between the Magellanic Clouds and the Milky Way (Mathewson et al. 1974). 
And third, other HVCs with low metallicities of $\sim 0.1$ solar have very likely an extragalactic - dwarf or intergalactic - origin. Blitz et al. (1999) suggested that some HVCs may represent material that failed to form galaxies. This material would be in gravitationally-bound systems, where most of the gravity would be provided by dark matter. These "mini-halos" would collect into filaments, the nearby ones falling into the Local Group. HVCs might then play a major role in the galaxy formation process. Explanations for the origins of IVCs mirror those offered for the HVCs, since there are many reasons to consider the origins of HVCs and IVCs together. Most of the IVCs fall into the "Galactic fountain" category (Richter et al. 2003b), because they appear to be located between 0.3 and $2.1 \mathrm{kpc}$ away from the Galactic plane (Wakker 2001) and have roughly solar metallicities.

Access to the total mass content of IVCs and HVCs is another key piece of information that can help pin down their origin. The vast majority of our knowledge on these clouds comes from $21 \mathrm{~cm}$ H I surveys, but what is their molecular content? For HVCs with an extragalactic origin, the molecular gas may be a possible counterpart of their dark matter content. The higher and higher angular resolution $\mathrm{H}$ I maps have shown considerable structure in HVCs down to the scale of the resolution (Schwarz \& Oort 1981; Wakker \& Schwarz 1991; Wakker et al. 2002). Wakker et al. (2001) found that this cloud structure is hierarchical and has structural similarities: the brightest knots are embedded in the brightest parts of the smoother background structure. This is the basic feature of fractals which allows a large amount of mass in the knots/cores within the clouds to remain hidden. The concept of fractals was first applied to HVC maps by Vogelaar \& Wakker (1994). The resolved H I cores within the HVCs have sizes down to at least 1 arcmin, H I column densities of a few times $10^{20} \mathrm{~cm}^{-2}$, brightness temperatures of up to $30 \mathrm{~K}$, and velocity widths of typically $\sim 8-10 \mathrm{~km} \mathrm{~s}^{-1}$. They hence appear to consist of cold gas, and most of the velocity width seen at lower resolution is due to random motions of these cores. The central densities in these cores can reach $>80 D_{\mathrm{kpc}}^{-1} \mathrm{~cm}^{-3}$, where $D_{\text {kpc }}$ is the distance of the HVC in kpc. Depending on the distance, these central densities may be high enough to form molecular gas.

The search for molecular gas in IVCs and HVCs was not very successful before UV measurements became possible. Indeed, except for two IVCs, the Draco Cloud (Mebold et al. 1985) and IV 21 (Weiss et al. 1999; Heithausen et al. 2001), which are observed in the disk-halo interface at less than $500 \mathrm{pc}$ and may well be "normal" molecular clouds at exceptionally large $z$ heights, no $\mathrm{CO}$ emission or absorption has been detected in Galactic halo clouds so far. Wakker et al. (1997) attempted to observe the $\mathrm{CO}$ emission toward six dense HVC cores, but no $\mathrm{CO}$ emission was detected down to a best $5 \sigma$ limit of $0.077 \mathrm{~K} \mathrm{~km} \mathrm{~s}^{-1}$ in their observations done with the NRAO $12 \mathrm{~m}$ telescope at Kitt Peak at an angular resolution of 1 arcmin. Similarly, Combes \& Charmandaris (2000) used the absorption technique toward 27 quasars known to be strong millimetric continuum sources, and obtained only one tentative detection of $\mathrm{HCO}^{+}$in a HVC. Molecular gas was found for the first time in $\mathrm{HVC}$ gas in absorption through the $\mathrm{H}_{2}$ electronic Lyman and Werner bands toward UV-bright background sources by Richter et al. (1999). Further $\mathrm{H}_{2}$ detections in absorption followed with the advent of FUSE in HVCs (Richter et al. 2001a,b; Sembach et al. 2001; Wakker 2006) and in IVCs, half of which currently show the presence of $\mathrm{H}_{2}$ (Richter et al. 2003b; Wakker 2006; Gillmon et al. 2006).
Additional strong evidence for molecular gas in HVCs was recently provided by Miville-Deschênes et al. (2005, hereafter MD05). They demonstrated the first detection of dust emission in the HVCs located on the edge of Complex $\mathrm{C}$ by comparing $21 \mathrm{~cm}$ data from the Green Bank Telescope (GBT) with very sensitive infrared observations from the Spitzer Space Telescope and from IRIS (Improved Reprocessing of the IRAS Survey, Miville-Deschênes \& Lagache 2005). They found clear correlations between $\mathrm{HI}$ and the infrared emission at 24, 60, 100 , and $160 \mu \mathrm{m}$. Previously dust emission was unsuccessfully looked for in HVCs using the Infrared Astronomical Satellite (IRAS) data (Wakker \& Boulanger 1986). The detected dust is found to be significantly colder, $T_{\mathrm{HVC}}=10.7_{-0.8}^{+0.9} \mathrm{~K}$, than in the local interstellar medium, $T_{\text {ISM }}=17.5 \mathrm{~K}$, in accordance with its association with cold gas. This dust emission, in addition, suggests a total column density of gas more than 5 times higher than the observed HI. This cold gas component, presumably in the form of molecular hydrogen, may thus be an important contribution to the total mass of HVCs.

We further investigate the molecular content of HVCs, presenting a new survey for $\mathrm{CO}$ emission performed with the IRAM $30 \mathrm{~m}$ telescope toward two main HVC HI cores of Complex $\mathrm{C}$ for which the dust emission was detected by MD05. Relative to the study by Wakker et al. (1997), we benefit from improved sensitivity, plus three times better angular resolution. This allowed us to explore the expected clumpy structure of the molecular gas, since the signal from the molecular clumps embedded in the H I cores is less diluted and the probability of detecting the $\mathrm{CO}$ emission is increased. In Sect. 2 we describe our observations and in Sect. 3 we present the results. The implications of this new $\mathrm{CO}$ emission survey in HVCs are discussed in Sect. 4, before concluding in Sect. 5 .

\section{Observations and data reduction}

The straightforward consequence of the discovery of cold dust emission from HVCs in Complex C by MD05 is a search for molecular gas via the $\mathrm{CO}$ emission. The Complex $\mathrm{C}$ spans some 1500 degrees squared on the sky. It has an average metallicity of 0.1-0.3 solar (Tripp et al. 2003; Collins et al. 2003, 2007) and is located at a distance greater than $5 \mathrm{kpc}$ from the Sun (Wakker 2001). The Spitzer Extragalactic First Look Survey (XFLS) field analyzed by MD05 covers only a $3^{\circ} \times 3^{\circ}$ area on the edge of Complex $\mathrm{C}$ centered at RA $=17 \mathrm{~h} 18 \mathrm{~m}$, Dec $=$ $+59^{\circ} 30 \mathrm{~m}(\mathrm{~J} 2000)$.

To select the most promising HVC regions for $\mathrm{CO}$ emission detection in this $3^{\circ} \times 3^{\circ}$ area, we first carefully re-examined the results obtained by MD05 to check whether we could spatially identify the infrared dust emission peaks in the HVCs. Using the public IRIS maps (Miville-Deschênes \& Lagache 2005) and the GBT $21 \mathrm{~cm} \mathrm{H}$ I data of the XFLS field kindly made available by Lockman \& Condon (2005), we extracted, as in MD05, the dust emission component corresponding to the HVCs from the decomposition of the infrared signal into different $\mathrm{H}$ I components identified in the $21 \mathrm{~cm}$ spectrum, namely the HVCs, two IVCs, and the local ISM H I emission (Fig. 4 in MD05). We confirm the global infrared-H I correlation found by MD05, but no spatial information on the HVC infrared emission can be derived. Indeed, the HVC infrared signal is spatially detected only at $2-3 \sigma$ (with a higher signal-to-noise ratio at larger scales), which makes reliable spatial identifications of infrared emission maxima difficult.

Therefore, we decided to choose the hopefully suitable HVC regions for our $\mathrm{CO}$ observations on the basis of $\mathrm{HI}$ core selection, corresponding to a HI column density maxima 

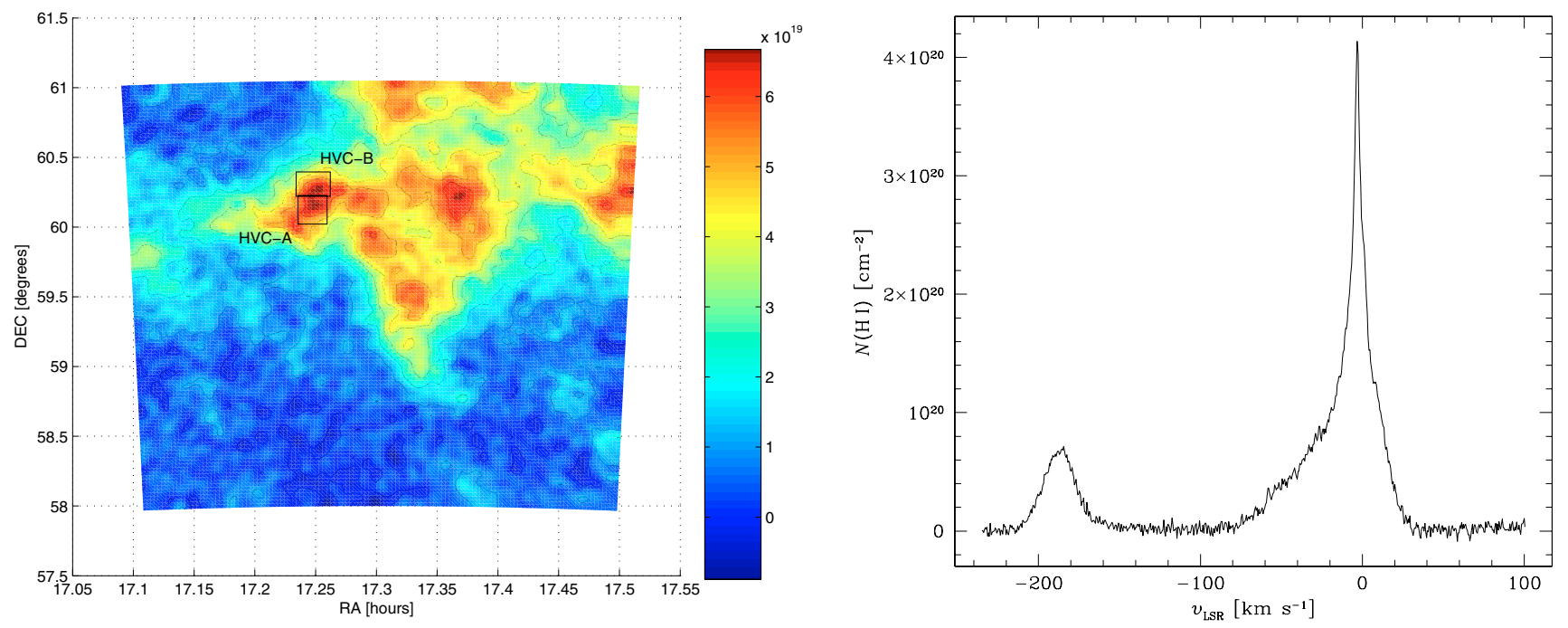

Fig. 1. Left. H I column density map in units of $\mathrm{cm}^{-2}$ of the HVCs in Complex $\mathrm{C}$ within the XFLS field. The map is obtained from the public GBT $21 \mathrm{~cm}$ data (Lockman \& Condon 2005). The HVCs are well isolated in space and their integrated emission was computed by adding all the channels between $-210<v_{\mathrm{LSR}}<-132 \mathrm{~km} \mathrm{~s}^{-1}$. The selected regions for the CO observations are the two framed H I cores labeled HVC-A and HVC-B, respectively. They correspond to two maxima of the HVC H I emission with column densities of $\sim 7 \times 10^{19} \mathrm{~cm}^{-2}$, and are the most promising regions for CO detection in these HVCs. Right. Average H I spectrum in the direction of the two selected cores. It clearly shows the contribution of the local ISM and of the HVC H I emissions.

selection. Such a strategy is supported by the recent results of Wakker (2006) which indicate that the fraction of sight lines with $\mathrm{H}_{2}$ detections in $\mathrm{UV}$, increases with $N(\mathrm{HI})$ for the IVCs. On the left panel of Fig. 1, we show the $21 \mathrm{~cm}$ emission map of the $3^{\circ} \times 3^{\circ}$ field analyzed by MD05, integrated over the HVC velocity channels $-210<v_{\text {LSR }}<-132 \mathrm{~km} \mathrm{~s}^{-1}$. We focused on two HVC HI cores: the HVC-A core located at $(\mathrm{J} 2000) \mathrm{RA}=17 \mathrm{~h} 14 \mathrm{~m} 59.055 \mathrm{~s}$, Dec $=+60^{\circ} 08 \mathrm{~m} 45.84 \mathrm{~s}$ and

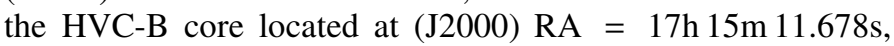
Dec $=+60^{\circ} 15 \mathrm{~m} 03.56 \mathrm{~s}$, both having among the larger $\mathrm{HI}$ column densities with $N(\mathrm{HI}) \sim 7 \times 10^{19} \mathrm{~cm}^{-2}$. The average $21 \mathrm{~cm}$ spectrum in the direction of the two cores is shown on the right panel of Fig. 1. We clearly see the contribution of the dominant local ISM H I emission and of the HVC H I emission at the LSR velocity of about $-190 \mathrm{~km} \mathrm{~s}^{-1}$.

The observations were performed with the IRAM $30 \mathrm{~m}$ millimeter-wave telescope at Pico Veleta, Spain, on June 20-23, 2006 under good weather conditions. We used four receivers simultaneously, two centered on the ${ }^{12} \mathrm{CO}(1-0)$ line at $115 \mathrm{GHz}$ and two on the ${ }^{12} \mathrm{CO}(2-1)$ line at $230 \mathrm{GHz}$. The beam width of the telescope at these two frequencies is $22^{\prime \prime}$ and $11^{\prime \prime}$, respectively. The data were recorded using the VESPA autocorrelator with $480 \mathrm{MHz}$ bandwidth and $0.32 \mathrm{MHz}$ resolution at $3 \mathrm{~mm}$ and two $1 \mathrm{MHz}$ filter banks (512 channels each) at $1 \mathrm{~mm}$. The resulting velocity coverage at $115 \mathrm{GHz}$ is $1250 \mathrm{~km} \mathrm{~s}^{-1}$ with $0.8 \mathrm{~km} \mathrm{~s}^{-1}$ resolution. The corresponding values at $230 \mathrm{GHz}$ are $660 \mathrm{~km} \mathrm{~s}^{-1}$ and $1.3 \mathrm{~km} \mathrm{~s}^{-1}$. To sample significant probability to find the $\mathrm{CO}$ molecular emission, we made $5 \times 5=25$ points maps centered on each of the two selected $\mathrm{HVC}$ regions. The observations followed the sequence from $-24^{\prime \prime}$ to $+24^{\prime \prime}$ offsets relative to the central coordinates of the HVC HI cores with a $12^{\prime \prime}$ spatial sampling in the right ascension direction and same in the declination direction, leading to $1^{\prime} \times 1^{\prime}$ maps. Spectra were obtained using the wobbler switch technique with a wobbler throw of $90^{\prime \prime}$. The total integration time on each point of the HVC-A and HVC-B maps varied between 9 and $26 \mathrm{~min}$, with a final observing time of $\sim 13 \mathrm{~h}$ on the sources.
The data were reduced with the CLASS software from the GILDAS package. The expected average CO line width is $\sim 10 \mathrm{~km} \mathrm{~s}^{-1}$, thus we first Hanning smoothed all the raw spectra at $3 \mathrm{~mm}$ to a resolution of $\sim 3 \mathrm{~km} \mathrm{~s}^{-1}$, assuming that a good line sampling requires at least 3 points. This leads to an increase of the signal-to-noise ratio per spectrum by a factor of 1.6. We then co-added all the smoothed spectra obtained with the two receivers tuned on the ${ }^{12} \mathrm{CO}(1-0)$ line and corresponding to the scans obtained at a given point of the HVC-A and HVC-B maps. We finally get a mosaic of 25 spectra centered on each of the selected HVC H I cores (Figs. 2 and 3). No baseline subtraction was done on individual spectra before the co-addition, since we perform a linear sum.

\section{Results}

In Figs. 2 and 3 we show the constructed mosaics of $25{ }^{12} \mathrm{CO}(1-0)$ spectra, centered on the two selected HVC H I cores for which cold dust emission was recently detected by MD05. These spectra show clearly that reliable molecular emission is not detected toward any position of the maps. We do observe a few tentative $\mathrm{CO}$ line detections with a significance level of $2-3 \sigma$ : for instance, the spectra at the offset positions $\left(-24^{\prime \prime} \times+24^{\prime \prime}\right),\left(0^{\prime \prime} \times-12^{\prime \prime}\right),\left(+12^{\prime \prime} \times-24^{\prime \prime}\right)$ and $\left(+24^{\prime \prime} \times-24^{\prime \prime}\right)$ in the HVC-A region, and $\left(-12^{\prime \prime} \times 0^{\prime \prime}\right)$ and $\left(0^{\prime \prime} \times-24^{\prime \prime}\right)$ in the HVC-B region. However, such spikes are very common in millimeter observations, occur randomly and result only from noise effects. Moreover, the LSR velocities of these tentative detections differ by 10 to $90 \mathrm{~km} \mathrm{~s}^{-1}$ from the expected LSR velocity of the two selected HVC H I cores as measured from $21 \mathrm{~cm}$ data.

Despite the lack of firm CO emission detection in our data, we can at least derive an upper limit on the molecular content in these HVCs which already provides interesting constraints. The baseline rms of our ${ }^{12} \mathrm{CO}(1-0)$ spectra vary between 11 and $24 \mathrm{mK}$ according to the different number of scans obtained at the various positions of the maps (see Figs. 2 and 3 for more details), with a mean rms value of $14.8 \mathrm{mK}$ for the HVC-A region and $15.4 \mathrm{mK}$ for the HVC-B region. 

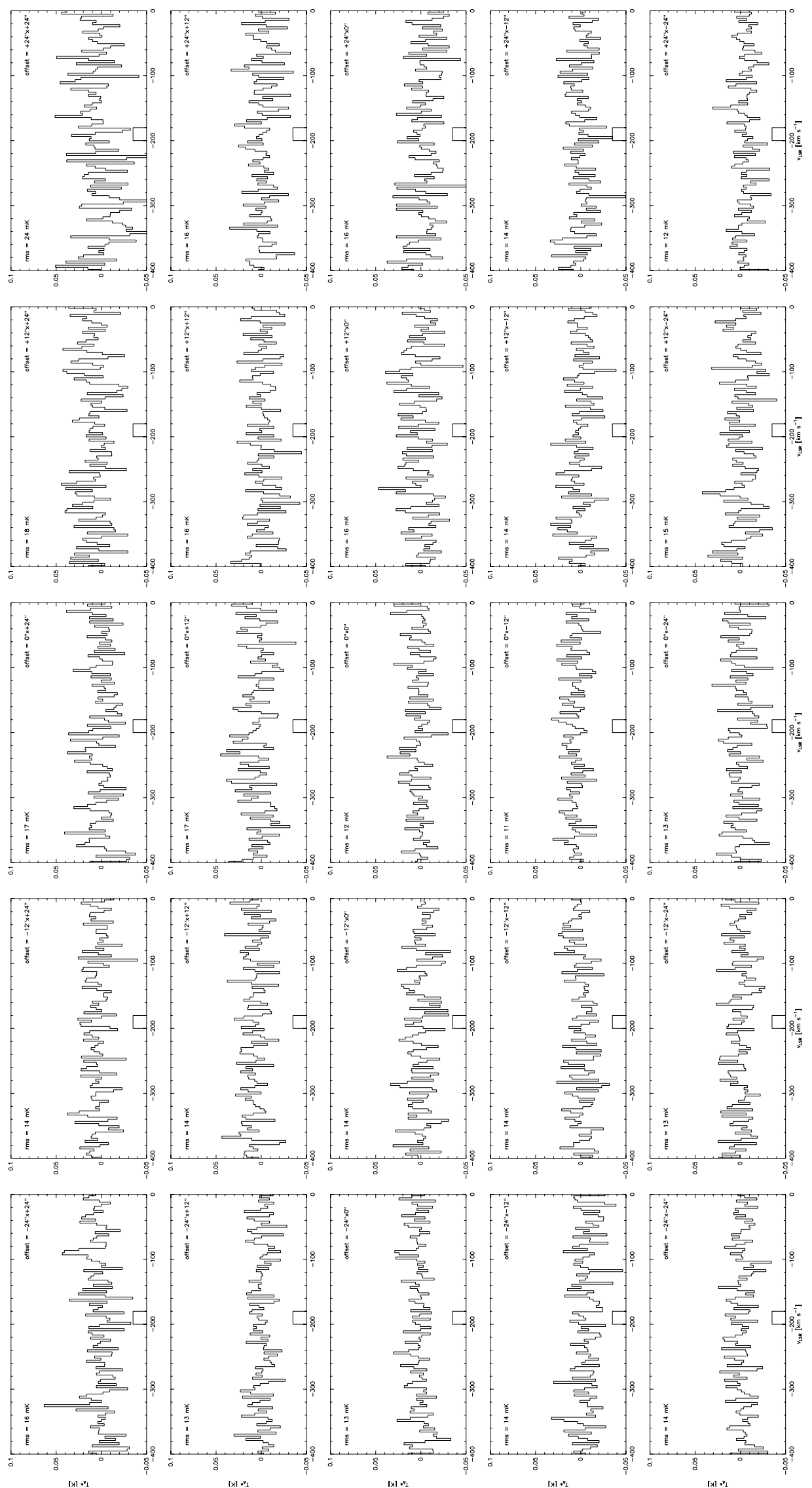

Fig. 2. Mosaic of $25^{12} \mathrm{CO}(1-0)$ spectra, covering a $60^{\prime \prime} \times 60^{\prime \prime}$ grid with a $12^{\prime \prime}$ spacing, centered on the H I core HVC-A. The spectra are smoothed to a resolution of $\sim 3 \mathrm{~km} \mathrm{~s}^{-1}$ and are the result of the co-addition of all scans obtained at a given position of the mosaic. The box shows the expected LSR velocity of the $\mathrm{CO}$ emission line according to the $21 \mathrm{~cm}$ data. 

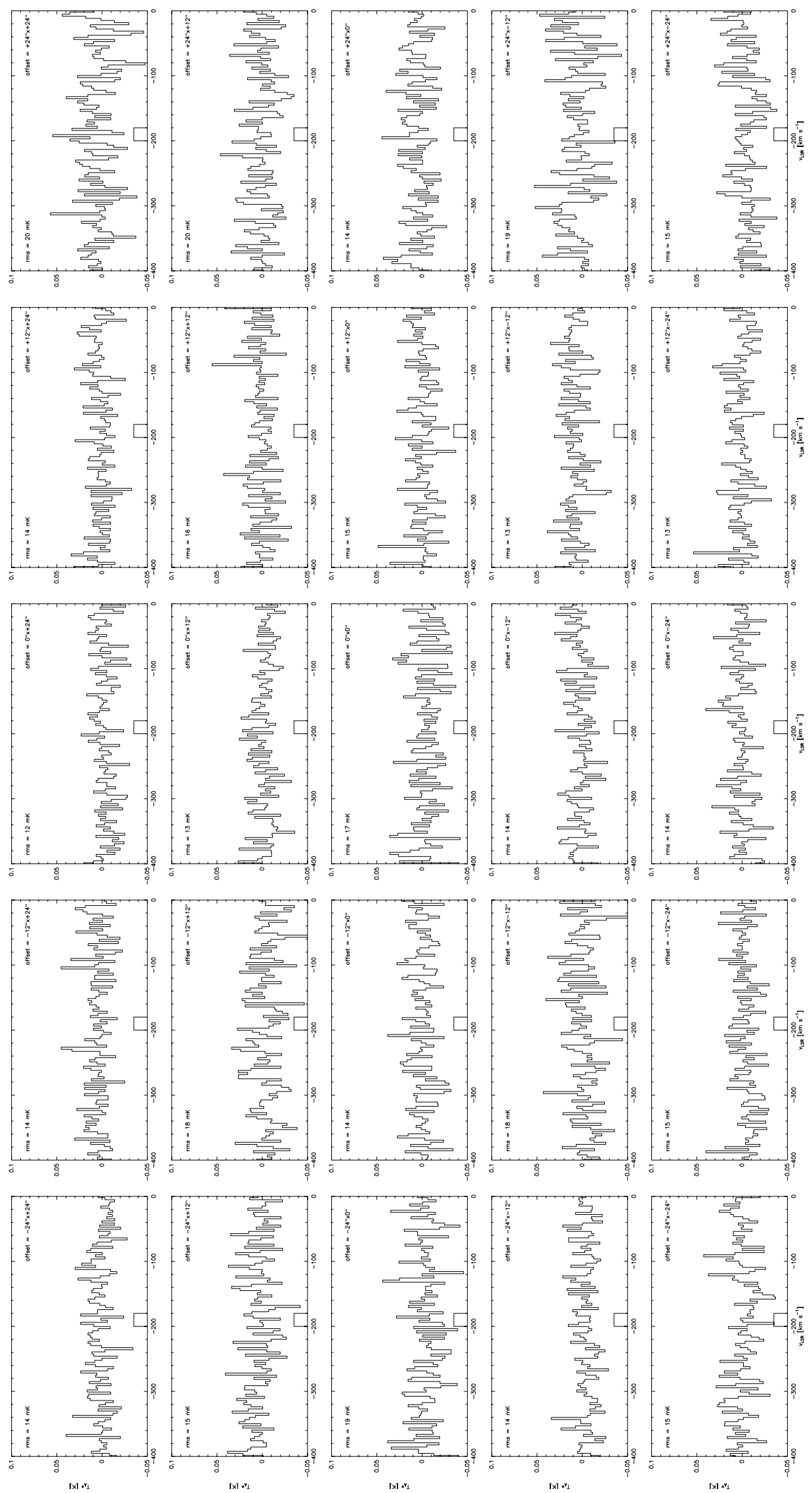

Fig. 3. Same as Fig. 2, but the mosaic is centered on the H I core HVC-B. 
Assuming a Gaussian line with $F W H M$ of $3 \mathrm{~km} \mathrm{~s}^{-1}$ (similar to Wakker et al. 1997, and see also Sect. 4.2), the mean rms values lead to average $5 \sigma \mathrm{CO}$ intensity detection limits of $I(\mathrm{CO})<0.22 \mathrm{~K} \mathrm{~km} \mathrm{~s}^{-1}$ and $0.23 \mathrm{~K} \mathrm{~km} \mathrm{~s}^{-1}$, respectively, in $22^{\prime \prime}$ beams. The most sensitive limit is derived toward the position $\left(0^{\prime \prime},-12^{\prime \prime}\right)$ of the HVC-A map with $I(\mathrm{CO})<0.16 \mathrm{~K} \mathrm{~km} \mathrm{~s}^{-1}$ (baseline $\mathrm{rms}=11 \mathrm{mK}$ ). Using the $\mathrm{CO}$-to- $\mathrm{H}_{2}$ conversion factor $X(\mathrm{CO})=1.9 \times 10^{20} \mathrm{~cm}^{-2}\left(\mathrm{~K} \mathrm{~km} \mathrm{~s}^{-1}\right)^{-1}$ determined by Strong $\&$ Mattox (1996) from the diffuse Galactic $\gamma$-ray emission, we get from the most sensitive observations the $5 \sigma \mathrm{H}_{2}$ column density detection limit of $N\left(\mathrm{H}_{2}\right)<3 \times 10^{19} \mathrm{~cm}^{-2}$.

In the case that the molecular gas is diffuse rather than clumpy, the co-addition of all the ${ }^{12} \mathrm{CO}(1-0)$ spectra of each HVC map is justified in order to obtain a CO intensity measurement over a larger beam hence equivalent to a $70^{\prime \prime}$ beam. The co-addition of spectra of the 25 positions leads to very good baseline rms of 2.9 and $3.0 \mathrm{mK}$ for the HVC-A and HVC-B regions, respectively, which correspond to a $5 \sigma \mathrm{CO}$ intensity detection limit of $I(\mathrm{CO})<0.04 \mathrm{~K} \mathrm{~km} \mathrm{~s}^{-1}$. This limit can then be directly compared with the upper limit $I(\mathrm{CO})<0.077 \mathrm{~K} \mathrm{~km} \mathrm{~s}^{-1}$ derived by Wakker et al. (1997), since it was determined over a similar beam size. We improved the sensitivity of the $\mathrm{CO}$ observations by a factor of 2 , but this is still not sufficient for detecting $\mathrm{CO}$ emission in HVCs.

\section{Discussion}

We collected a new set of CO observations toward two H I cores of HVCs in Complex $\mathrm{C}$ for which cold dust emission was recently detected by MD05. Although our data have a three times better angular resolution and a two times better sensitivity than those of Wakker et al. (1997), no firm CO emission is detected. How can we reconcile this persistent non-detection of $\mathrm{CO}$ emission in HVCs with the possible presence of cold dust as suggested by MD05 and with the recent FUSE results in IVCs and HVCs?

\subsection{Direct implications of the CO non-detection}

Half of the investigated IVCs with FUSE show the presence of molecular hydrogen in absorption (Richter et al. 2003b; Wakker 2006; Gillmon et al. 2006). The total $\mathrm{H}_{2}$ column densities measured are $\log N\left(\mathrm{H}_{2}\right)=14.1-16.4$. This widespread existence of $\mathrm{H}_{2}$ with low molecular fractions, $\log f$, varying between -1.4 (upper limits) and -5.3 (measured values) and low volume densities estimated to be $n_{\mathrm{H}}=10-50 \mathrm{~cm}^{-3}$ from the $\mathrm{H}_{2}$ formationdissociation equilibrium, very likely traces the cold, diffuse medium of the halo clouds, because of its large filling factor (Richter et al. 2003b). Indeed, the chance of detecting such a diffuse $\mathrm{H}_{2}$ absorber by way of absorption spectroscopy toward a limited number of background sources is much higher than that of finding a small, dense clump which would have a small angular extent: for a 10 times higher volume density, the linear size of the molecular clouds is 10 times smaller, if the H I column density stays constant; and thus, the filling factor is already 100 times lower when assuming a spherical geometry.

The sightlines passing through HVCs do not seem to show the same ubiquity of $\mathrm{H}_{2}$ as the IVCs. Indeed, $\mathrm{H}_{2}$ was found toward the Magellanic Stream with $\log N\left(\mathrm{H}_{2}\right)=16.4-18.2$ (Sembach et al. 2001; Richter et al. 2001b; Wakker 2006), but no $\mathrm{H}_{2}$ is for instance detected in Complex $\mathrm{C}$ down to limits of $\log N\left(\mathrm{H}_{2}\right)<13.8$ (Richter et al. 2001b; Wakker 2006). This shows that the low FUV radiation field, at least as weak as in the IVCs reducing the UV photodissociation of $\mathrm{H}_{2}$, and the relatively high $\mathrm{H}$ I column densities sampled of up to $10^{20} \mathrm{~cm}^{-2}$ are not sufficient to allow diffuse $\mathrm{H}_{2}$ to exist at moderate densities. The $\sim 0.1$ sub-solar metallicity and the abundance patterns measured in Complex C (Tripp et al. 2003; Collins et al. 2003, 2007) indicating a low dust content led Richter et al. (2003b) to conclude that the $\mathrm{H}_{2}$ formation on the surface of dust grains working as catalysts must be suppressed and that the alternative gas phase $\mathrm{H}_{2}$ formation processes (Black 1978) are not efficient enough to enable diffuse molecular gas.

The non-detection of diffuse $\mathrm{H}_{2}$ together with the nondetection of $\mathrm{CO}$ emission despite a two-fold better sensitivity reached in our observations in comparison with the Wakker et al. (1997) data do not provide at a first glance any evidence that the HVCs in Complex $\mathrm{C}$ contain large amounts of molecular gas. Hence these results do not confirm the findings of MD05 and bring into question the reliability of their cold dust emission detection.

A close inspection of the MD05 analysis indicates that the derived contribution of the HVCs to the infrared emission is at the level of the residual term they find from the decomposition of the measured infrared signal into the different Hi components identified in the $21 \mathrm{~cm}$ observations, namely the HVCs, two IVCs, and the local Galactic ISM (see Table 1 in MD05). The determination of the dust component of the HVCs is thus strongly dependent on the fit of the total infrared emission. In particular, the cold nature of the dust in the HVCs is crucially dependent on the fit of the dust emission at $100 \mu \mathrm{m}$, with a derived HVC emissivity 10 times smaller than that of the local ISM gas, against only a factor of 2 smaller at $160 \mu \mathrm{m}$. The interpretation of Complex $\mathrm{C}$ infrared data by MD05 raises incertainties. Their results thus require confirmation by additional measurements.

\subsection{The complexity of the physical conditions at play}

The situation, however, is even more complex and different configurations can explain the non-detection of both the $\mathrm{CO}$ emission and the diffuse $\mathrm{H}_{2}$ without implying the absence of molecular gas in the HVCs of Complex C. The presence of cold dust emission as supported by MD05 and the lack of CO emission may thus well be compatible.

Molecular clumps with high volume densities certainly exist in HVCs, but they are not traced by the diffuse molecular gas component. These clumps should contain molecular hydrogen at a high fractional abundance, since the dissociating FUV field in the halo clouds is not intense and the $\mathrm{H}_{2}$ formation timescale becomes shorter at high densities $\left(t_{\text {form }} \propto n_{\mathrm{H}}^{-1}\right)$. Our $\mathrm{CO}$ observations with a detection limit 5 orders of magnitude higher than that of $\mathrm{H}_{2}$ absorption studies, precisely aim to detect the emission peaks of these clumps. It has, indeed, been demonstrated over the last few years that the cold neutral component of the ISM consists of substantial small-scale structures at $\mathrm{AU}$ scales, from $1 \mathrm{pc}$ down to $10 \mathrm{AU}$ (the resolution limit of the current observations), indicating ubiquitous tiny $\mathrm{H}$ I gaseous clumps with temperatures as low as $50 \mathrm{~K}$ and very high densities, $n_{\mathrm{H}} \sim 10^{3}-10^{5} \mathrm{~cm}^{-3}$ (e.g. Faison et al. 1998; Lauroesch et al. 2000). Recently, the CO emission of such tiny molecular clumps with sizes of a few hundred $\mathrm{AU}$, temperatures between 7 and $18 \mathrm{~K}$, volume densities of a few $10^{4} \mathrm{~cm}^{-3}$, and column densities of $\log N\left(\mathrm{H}_{2}\right)=19-21$ have been observed in cirrus clouds and have revealed that these clumps are fractally structured (Heithausen 2004, 2006).

Such AU-scale molecular clumps could have easily been diluted in Wakker et al. (1997) observations made at an angular 
resolution of $1^{\prime}$ in contrast to the $3^{\prime \prime}$ resolution data of Heithausen, and have therefore been undetected. Our new survey for $\mathrm{CO}$ emission in HVCs benefits from a three times better angular resolution. The size of the clumps resolved in our $22^{\prime \prime}$ beam width hence is three times lower, namely $0.5 \mathrm{pc}$ when assuming that the HVCs in Complex $\mathrm{C}$ are about $5 \mathrm{kpc}$ away from the Galactic plane ${ }^{1}$ (Wakker 2001). A 0.5 pc resolution sampled by the observations is within the range of reported sizes of molecular clouds, and hence our observations should be less affected by the dilution effect. However, we still do not detect the $\mathrm{CO}$ emission in HVCs. This suggests that the dilution problem remains for the very dense clumps. In the absence of agitation and heating by star formation, the fraction of dense and thus small clumps can indeed be higher. Assuming that the HVCs have a similar size distribution of molecular clumps as the cirrus clouds (Heithausen 2004), $\mathrm{H}_{2}$ column densities higher than our detection limit, $N\left(\mathrm{H}_{2}\right)=3 \times 10^{19} \mathrm{~cm}^{-2}$, are hence expected to be confined in very small clumps with 20 times smaller sizes than our $0.5 \mathrm{pc}$ resolution. Although present, they thus are still highly diluted in our observations made with the IRAM 30 m telescope.

Moreover, assuming the same fractal structure for molecular clouds in the HVCs as that traced by the size-line width relation (e.g. Solomon et al. 1987), we expect a series of clumps of all sizes in our beam. The global ensemble is composed of molecular clumps of the order of $1 \mathrm{pc}$ size (the resolution scale) and with a dispersion in velocity $\sigma_{v} \sim 1 \mathrm{~km} \mathrm{~s}^{-1}$ or a $F W H M$ of about $3 \mathrm{~km} \mathrm{~s}^{-1}$. It is only at that size scale that we detect resolved clumps which hence have an average surface density lower than $3 \times 10^{19} \mathrm{~cm}^{-2}$ as derived from the $\mathrm{CO}$ emission detection limit, or a volume density lower than $20 \mathrm{~cm}^{-3}$, at $5 \sigma$. This is much lower than the minimal density required to excite the $\mathrm{CO}$ molecules which is about $10^{3} \mathrm{~cm}^{-3}$. As a consequence, the inter-clump gas already at the $1 \mathrm{pc}$ scale appears to be too diffuse to excite the $\mathrm{CO}$ molecules.

Alternatively, the non-detection of $\mathrm{CO}$ emission might be ascribed to the peculiar physical conditions that reign in the HVCs of Complex C. First, the sub-solar metallicity of $0.1-0.3 \mathrm{dex}$ measured by Tripp et al. (2003) and Collins et al. (2003, 2007) in these HVCs might be decisive. As stated in Sect. 4.1, Richter et al. (2003b) have invoked the argument that the lower dustto-gas ratio associated with low metallicity media leads to the suppression of the most efficient $\mathrm{H}_{2}$ formation process onto dust grains in order to explain the non-detection of diffuse $\mathrm{H}_{2}$ in Complex C. However, the unknown dust properties in HVCs, e.g. the grain size distribution and the grain surface, make the implications of low metallicities on the $\mathrm{H}_{2}$ grain formation rate very uncertain. It has, in addition, been empirically shown that the lack of heavy elements also appears to directly affect the relationship between the tracer molecule $\mathrm{CO}$ and $\mathrm{H}_{2}$ in the way that large quantities of $\mathrm{H}_{2}$ molecular gas are not necessarily traced by large $\mathrm{CO}$ emission. In low metallicity media, an underabundance of dust may result in more intense radiation fields, and therefore while $\mathrm{H}_{2}$ survives because of self-shielding, this does not stop photodissociation of CO (e.g. Maloney \& Black 1988).

Leroy et al. (2007) recently derived a new estimation of the CO-to- $\mathrm{H}_{2}$ conversion factor over the entire Small Magellanic Cloud (SMC), characterized by a low metallicity similar to that of the HVCs in Complex C. They found $X(\mathrm{CO})=13 \times 10^{21} \mathrm{~cm}^{-2}\left(\mathrm{~K} \mathrm{~km} \mathrm{~s}^{-1}\right)^{-1}$ in agreement with the value previously obtained by Israel (1997) in the SMC, namely about 60 times higher than the conversion factor

1 If the HVCs in Complex $\mathrm{C}$ are at a greater distance than $5 \mathrm{kpc}$ from the Galactic plane, the resolved size of the clumps is less than $0.5 \mathrm{pc}$.
$X(\mathrm{CO})=1.9-2.3 \times 10^{20} \mathrm{~cm}^{-2}\left(\mathrm{~K} \mathrm{~km} \mathrm{~s}^{-1}\right)^{-1}$ determined for molecular clouds in the Galactic plane with approximately solar metallicities (Bloemen et al. 1989; Strong \& Mattox 1996). This implies that for the same $\mathrm{H}_{2}$ column density the corresponding integrated intensity of $\mathrm{CO}$ is 60 times lower in low metallicity than in solar metallicity molecular clouds. As a consequence, the detection of molecular gas in low metallicity HVCs suffers from the low $\mathrm{CO}$ intensity, and may result in a drastic lack of sensitivity for detecting $\mathrm{CO}$ emission despite a possibly large amount of $\mathrm{H}_{2}$ molecular gas in HVCs. This can perfectly reconcile the presence of cold dust emission as claimed by MD05 and the CO emission non-detection. MD05 estimated the total gas column density of the HVCs to be more than 5 times higher than observed in $\mathrm{HI}$. This cold gas $\left(T_{\mathrm{HVC}}=10.7 \mathrm{~K}\right)$ component if entirely associated with molecular gas implies a high $\mathrm{H}_{2}$ column density of at least $N\left(\mathrm{H}_{2}\right) \approx 3.5 \times 10^{20} \mathrm{~cm}^{-2}$. Using the CO-to- $\mathrm{H}_{2}$ conversion factor for SMC metallicity molecular clouds, the corresponding $\mathrm{CO}$ intensity still is very weak, $I(\mathrm{CO}) \approx 0.027 \mathrm{~K} \mathrm{~km} \mathrm{~s}^{-1}$, namely one order of magnitude below our $\mathrm{CO}$ detection limit.

Secondly, the very low temperature of $T_{\mathrm{HVC}}=10.7_{-0.8}^{+0.9} \mathrm{~K}$ estimated in the HVCs of Complex $\mathrm{C}$ by MD05 from the infrared dust emission might have severe consequences. Indeed, solving a Clausius-Clapeyron type equation for the critical vapor pressure of $\mathrm{CO}$ ice sublimation $\ln P=a / T+b$ using the CO triple point $\left(T_{3}=68.14 \mathrm{~K}, P_{3}=0.1535 \times 10^{6} \mathrm{dyn}\right)$ and the CO critical point $\left(T_{\mathrm{c}}=132.91 \mathrm{~K}, P_{\mathrm{c}}=34.987 \times 10^{6} \mathrm{dyn}\right)^{2}$, we find $P=1.04 \times 10^{10} \exp (-759 / T)$ dyn. Below $20 \mathrm{~K}$ the CO molecule can be in a solid state even under interstellar medium pressure conditions. This implies that $\mathrm{CO}$ may condense onto dust grains and hence be depleted in gas-phase observations. Leger (1983) has examined this CO sublimation effect in dark molecular clouds, and current measurements in starless/pre-stellar cores show evidence for $\mathrm{CO}$ depletion by a factor of 10 (e.g. Bacmann et al. 2002; Tafalla et al. 2002). Similarly, such CO depletion levels can easily be expected in HVCs if the temperatures are at least as low as $20 \mathrm{~K}$ in these clouds.

\section{Conclusions}

The recent detection of cold dust emission in the HVCs of Complex $\mathrm{C}$ by MD05 brought a new surge of hope for the presence of large amounts of molecular gas in HVCs and its possible detection via $\mathrm{CO}$ emission. We thus undertook a survey for molecular gas toward the HVCs observed by MD05 with the IRAM $30 \mathrm{~m}$ telescope at an angular resolution of 22 arcsec. We obtained two mosaics of $25{ }^{12} \mathrm{CO}(1-0)$ spectra covering a $1^{\prime} \times 1^{\prime}$ area each and centered on two HVC H I cores with column densities $N(\mathrm{HI}) \sim 7 \times 10^{19} \mathrm{~cm}^{-2}$. No firm CO emission line at the HVC LSR velocity was detected. The best baseline rms value of $11 \mathrm{mK}$ obtained leads to a $5 \sigma \mathrm{CO}$ intensity detection limit of $I(\mathrm{CO})<0.16 \mathrm{~K} \mathrm{~km} \mathrm{~s}^{-1}$. The improved sensitivity and angular resolution by a factor of two and three, respectively, compared with the CO observations made by Wakker et al. (1997) in HVCs, show that this still is not sufficient for detecting the CO emission in HVCs.

The non-detection of both the $\mathrm{CO}$ emission and the diffuse $\mathrm{H}_{2}$ (Richter et al. 2001b; Wakker 2006) in the HVCs of Complex $\mathrm{C}$ do not provide at a first glance any evidence for large amounts of molecular gas in these clouds, and hence do not support the findings by MD05. Although the reliability of their

\footnotetext{
2 Encyclopédie des gaz, Gas Encyclopaedia, L'Air Liquide, 1976, Elsevier, Amsterdam.
} 
analysis may be questioned and requires confirmation by additional measurements, our negative $\mathrm{CO}$ detection does not, however, allow their results to be refuted, because the physical conditions at play are far too complex and still not well constrained. We can invoke different configurations which enable reconciliation of the $\mathrm{CO}$ emission non-detection with the presence of molecular gas and hence of cold dust emission:

(i) Our CO data may still suffer strong dilution effects:

The typical clouds resolved in the $22^{\prime \prime}$ beam width are of $0.5 \mathrm{pc}$ if the HVCs in Complex $\mathrm{C}$ are at a distance of about $5 \mathrm{kpc}$. They enter the size range observed in molecular clouds. However, taking at face value the Heithausen (2004) results obtained in cirrus clouds, the molecular clumps with $\mathrm{H}_{2}$ column densities higher than our detection limit, $N\left(\mathrm{H}_{2}\right)=$ $3 \times 10^{19} \mathrm{~cm}^{-2}$, should still have about 20 times smaller sizes compared to our resolution. This suggests that most of the molecular gas may be confined in very small and dense clumps in HVCs which are then highly diluted in our observations.

(ii) The $\mathrm{CO}$ non-detection may be the result of a too diffuse interclump medium, filling a typical molecular cloud, to excite the CO molecules:

The global ensemble of molecular clumps resolved in our beam are of the order of $1 \mathrm{pc}$. Since these clumps are undetected, they have an average surface density lower than our detection limit. This corresponds to a volume density lower than $20 \mathrm{~cm}^{-3}$ at $5 \sigma$, namely lower than the minimal density required for the $\mathrm{CO}$ excitation.

(iii) The sub-solar metallicity of $0.1-0.3$ dex measured in the HVCs of Complex C may play an important role:

Partly because the lower dust-to-gas ratio may lead to the suppression of the most efficient $\mathrm{H}_{2}$ formation process onto dust grains. And more specifically, because as Leroy et al. (2007) showed, the CO-to- $\mathrm{H}_{2}$ conversion factor measured at the SMC metallicity is 60 times higher than at the solar metallicity. This implies that for a given $\mathrm{H}_{2}$ column density, the corresponding integrated $\mathrm{CO}$ intensity will be 60 times lower in low metallicity molecular clouds. As a consequence, despite the large molecular gas content estimated by MD05 from the cold dust emission detected in the HVCs of Complex $\mathrm{C}$, the expected $\mathrm{CO}$ intensity is $I(\mathrm{CO}) \approx$ $0.027 \mathrm{~K} \mathrm{~km} \mathrm{~s}^{-1}$, namely one order of magnitude below our $\mathrm{CO}$ detection limit.

(iv) $\mathrm{CO}$ may be partly depleted in gas-phase observations: MD05 determined a very low temperature of $\sim 10.7 \mathrm{~K}$ for the dust in the HVCs of Complex C. If correct, this implies the presence of molecular gas cold enough to allow CO condensation onto dust grains under ISM pressure conditions.

These results illustrate the difficulty of determining the molecular gas content in the HVCs. The Atacama Large Millimeter Array, ALMA, should achieve at least a 10 times better sensitivity and angular resolution than the current millimeter instruments, and will hence hopefully satisfy the requirements for detecting $\mathrm{CO}$ emission in physical media with characteristics similar to those of HVCs. The determination of the physical conditions and origins of HVCs is crucial to better understand their implications in the formation and evolution of galaxies that may be in multiple forms, for example as gas fuel for star formation, as potential tracers of small dark matter halos, and as low metallicity gas for chemical evolution.

Acknowledgements. The authors wish to thank the IRAM $30 \mathrm{~m}$ staff for their valuable support and advice. M.D.-Z. is, in particular, grateful to Sergio Martín for allowing her to benefit of his large millimeter observation experience. M.D.-Z. also extends special thanks to Brad K. Gibson for helpful discussion on high-velocity clouds during his stay at Geneva Observatory.

\section{References}

Bacmann, A., Lefloch, B., Ceccarelli, C., et al. 2002, A\&A, 389, L6 Black, J. 1978, ApJ, 222, 125

Blitz, L., Spergel, D. N., Teuben, P. J., Hartmann, D., \& Burton, W. B. 1999, ApJ, 514, 818

Bloemen, H. 1989, ARA\&A, 27, 469

Collins, J. A., Shull, J. M., \& Giroux, M. L. 2003, ApJ, 585, 336

Collins, J. A., Shull, J. M., \& Giroux, M. L. 2007, ApJ, 657, 271

Combes, F., \& Charmandaris, V. 2000, A\&A, 357, 75

Faison, M. D., Goss, W. M., Diamond, P. J., \& Taylor, G. B. 1998, AJ, 116, 2916

Gibson, B. K., Giroux, M. L., Penton, S. V., et al. 2000, AJ, 120, 1830

Gibson, B. K., Giroux, M. L., Penton, S. V., et al. 2001, AJ, 122, 3280

Gillmon, K., Shull, J. M., Tumlinson, J., \& Danforth, C. 2006, ApJ, 636, 891

Hartmann, D., \& Burton, W. B. 1997, Atlas of Galactic Neutral Hydrogen (Cambridge University Press)

Heithausen, A. 2004, ApJ, 606, L13

Heithausen, A. 2006, A\&A, 450, 193

Heithausen, A., Weiss, A., Kerp, J., \& Fritz, T. 2001, ApJ, 561, 238

Houck, J. C., \& Bregman, J. N. 1990, ApJ, 352, 506

Israel, F. P. 1997, A\&A, 328, 471

Lauroesch, J. T., Meyer, D. M., \& Blades, J. C. 2000, ApJ, 543, L43

Leger, A. 1983, A\&A, 123, 271

Leroy, A., Bolatto, A., Stanimirovic, S., et al. 2007, ApJ, in press [arXiv:astro-ph/0611687]

Lockman, F. J., \& Condon, J. J. 2005, AJ, 129, 1968

Lu, L., Sargent, W. L. W., Savage, B. D., et al. 1998, AJ, 115, 162

Maloney, P., \& Black, J. H. 1988, ApJ, 325, 389

Mathewson, D. S., Cleary, M. N., \& Murray, J. D. 1974, ApJ, 190, 291

Mebold, U., Cernicharo, J., Velden, L., et al. 1985, A\&A, 151, 427

Miville-Deschênes, M.-A., \& Lagache, G. 2005, ApJS, 157, 302

Miville-Deschênes, M.-A., Boulanger, F., Reach, W. T., \& Noriega-Crespo, A. 2005, ApJ, 631, L57 (MD05)

Muller, C. A., Oort, J. H., \& Raimond, E. 1963, CR Acad. Sci. Paris, 257, 1661

Murphy, E., Lockman, F. J., \& Savage, B. D. 1995, ApJ, 447, 642

Oort, J. H. 1970, A\&A, 7, 381

Richter, P., de Boer, K. S., Widmann, H., et al. 1999, Nature, 402, 386

Richter, P., Savage, B. D., Wakker, B. P., Sembach, K. R., \& Kalberla, P. M. W. 2001a, ApJ, 549, 281

Richter, P., Sembach, K. R., Wakker, B. P., \& Savage, B. D. 2001b, ApJ, 562, L181

Richter, P., Sembach, K. R., Wakker, B. P., et al. 2001c, ApJ, 559, 318

Richter, P., Sembach, K. R., \& Howk, J. C. 2003a, A\&A, 405, 1013

Richter, P., Wakker, B. P., Savage, B. D., \& Sembach, K. R. 2003b, ApJ, 586, 230

Schwarz, U. J., \& Oort, J. H. 1981, A\&A, 101, 305

Shapiro, P. R., \& Field, G. B. 1976, ApJ, 205, 762

Sembach, K. R., Howk, J. C., Savage, B. D., \& Shull, J. M. 2001, AJ, 121, 992

Solomon, P. M., Rivolo, A. R., Barrett, J., \& Yahil, A. 1987, ApJ, 319, 730

Strong, A. W., \& Mattox, J. R. 1996, A\&A, 308, L21

Tafalla, M., Myers, P. C., Caselli, P., Walmsley, C. M., \& Comito, C. 2002, ApJ, 569,815

Tripp, T. M., Wakker, B. P., Jenkins, E. B., et al. 2003, AJ, 125, 3122

van Woerden, H., Schwarz, U. J., Peletier, R. F., Wakker, B. P., \& Kalberla, P. M. W. 1999, Nature, 400, 138

van Woerden, H., Wakker, B. P., Schwarz, U. J., \& de Boer, K. S. 2004, High Velocity Clouds (Dordrecht: Kluwer)

Vogelaar, M. G. R., \& Wakker, B. P. 1994, A\&A, 291, 557

Wakker, B. P. 2001, ApJS, 136, 463

Wakker, B. P. 2006, ApJS, 163, 282

Wakker, B. P., \& Boulanger, F. 1986, A\&A, 170, 84

Wakker, B. P., \& Schwarz, U. J. 1991, A\&A, 250, 484

Wakker, B. P., \& van Woerden, H. 1997, ARA\&A, 35, 217

Wakker, B. P., Murphy, E. M., van Woerden, H., \& Dame, T. M. 1997, ApJ, 488, 216

Wakker, B. P., Howk, J. C., Savage, B. D., et al. 1999, Nature, 402, 388

Wakker, B. P., Kalberla, P. M. W., van Woerden, H., de Boer, K. S., \& Putman, M. E. 2001, ApJS, 136, 537

Wakker, B. P., Oosterloo, T. E., \& Putman, M. E. 2002, AJ, 123, 1953

Weiss, A., Heithausen, A., Herbstmeier, U., \& Mebold, U. 1999, A\&A, 344, 955 Wolfire, M. G., McKee, C. F., Hollenbach, D., \& Tielens, A. G. G. M. 1995, ApJ, 453,673 